\title{
Grapheme-color associations can transfer to novel graphemes when synesthetic colors function as grapheme "discriminating markers"
}

\author{
Kyuto Uno ${ }^{1} \cdot$ Michiko Asano $^{2} \cdot$ Hana Kadowaki $^{3} \cdot$ Kazuhiko Yokosawa $^{1}$
}

Published online: 6 April 2020

(C) The Psychonomic Society, Inc. 2020

\begin{abstract}
Synesthesia is a condition in which the perception of a stimulus in one modality automatically triggers a secondary sensation in another modality or processing stream. Our study focused on grapheme-color synesthesia, in which the visual perception of letters or numbers (graphemes) induces a specific color sensation (the synesthetic color). Grapheme-color synesthetes do not typically experience colors for novel graphemes. However, synesthetic colors associated with familiar graphemes can be transferred to graphemes learned later, even in adulthood. A previous study has shown that such a transfer can take place after only a 10-min writing exercise. In this study, we found that this immediate transfer occurs only when the synesthetic colors for familiar graphemes contribute to the discrimination of the graphemes to be learned. Synesthetes learned six novel graphemes, each of which was arbitrarily associated with one of six familiar graphemes. Half of the synesthetes were assigned to the heterogeneous condition, in which the synesthetic colors of one group of familiar graphemes were different from one another. The other half of the synesthetes were assigned to the homogeneous condition, in which the various colors of a whole group of familiar graphemes were categorically the same. After this learning session, less transfer of synesthetic colors to novel graphemes from the corresponding familiar graphemes occurred in the homogeneous condition than in the heterogeneous condition. These results support the view that synesthetic colors for graphemes may function as a grapheme acquisition aid.
\end{abstract}

Keywords Synesthesia $\cdot$ Grapheme learning $\cdot$ Associative learning

\section{Introduction}

Synesthesia is a condition in which the perception of a stimulus in one modality (the inducer) automatically triggers a secondary sensation in another modality or processing stream (the concurrent). Our study focused on grapheme-color synesthesia, in which the visual perception of letters or numbers (graphemes) induces a specific color sensation (the synesthetic color). In

Electronic supplementary material The online version of this article (https://doi.org/10.3758/s13423-020-01732-9) contains supplementary material, which is available to authorized users.

Kyuto Uno

uno@1.u-tokyo.ac.jp

1 Department of Psychology, Graduate School of Humanities and Sociology, The University of Tokyo, Tokyo, Japan

2 Department of Psychology, College of Contemporary Psychology, Rikkyo University, Saitama, Japan

3 Department of Psychology, Faculty of Letters, The University of Tokyo, Tokyo, Japan each grapheme-color synesthete, the associations between graphemes and colors are highly consistent over time (Eagleman, Kagan, Nelson, Sagaram, \& Sarma, 2007; Rich, Bradshaw, \& Mattingley, 2005).

Synesthetes do not typically experience colors for novel graphemes (Mroczko, Metzinger, Singer, \& Nikolić, 2009). However, synesthetic colors associated with graphemes can be transferred to graphemes learned later, even in adulthood (Rich et al., 2005; Witthoft \& Winawer, 2006). For example, if an English-speaking synesthete who experiences the color purple for the English letter " $F$ " later learns the Russian language, the Cyrillic " $F$ " $(\Phi)$ may also become associated with the same purple color (Witthoft \& Winawer, 2006). Furthermore, Mroczko et al. (2009) demonstrated that such a transfer could take place after only a 10-min writing exercise. In their study, each synesthete learned three novel graphemes (Glagolitic graphemes) arbitrarily associated with familiar graphemes (Latin letters or Arabic digits) that initially produced strong grapheme-color associations. After spending less than $10 \mathrm{~min}$ on a writing exercise using the familiarnovel grapheme pairs, most synesthetes began to experience 
synesthetic colors for the novel graphemes, and the colors were identical to those that had been associated with the familiar graphemes. These studies showing synesthetic color transfer support the view that semantic decoding of the graphemes plays an important role in the process of grapheme-color synesthesia (Asano \& Yokosawa, 2012; Dixon, Smilek, Duffy, Zanna, \& Merikle, 2006; Mroczko et al., 2009; Simner, 2007). This semantic hypothesis (Mroczko et al., 2009) proposes that synesthetic grapheme-color associations emerge at the semantic/conceptual level rather than at the perceptual level of grapheme representation.

The semantic hypothesis (Mroczko et al., 2009) suggests that a synesthetic color associated with a grapheme can be automatically transferred to a novel grapheme as soon as the synesthete learns that the novel grapheme is semantically/ conceptually equivalent to the familiar one. However, considering recent discussions on the relationship between grapheme-color synesthesia and grapheme learning (Asano \& Yokosawa, 2013; Watson, Akins, Spiker, Crawford, \& Enns, 2014; Watson, Chromý, Crawford, Eagleman, Enns, \& Akins, 2017), the "discriminating" potential of the synesthetic color is one of the factors that may modulate whether synesthetic color transfer to a novel grapheme occurs. Synesthetic inducers often involve complex entities - for example, graphemes, numbers, days of the week, and musical notes, which children learn with effort (Watson et al., 2014). Such learning requires identification and discrimination of the members of the to-be-learned domain. A recent line of research on grapheme-color synesthesia and grapheme learning suggests that synesthetic colors may facilitate grapheme discrimination, and in turn grapheme learning, in childhood (Asano \& Yokosawa, 2013; Watson et al., 2014, 2017). Watson et al. (2017) conducted a large population study and revealed that grapheme-color synesthesia is more common among native speakers of languages with more difficult (i.e., orthographically opaque) writing systems, suggesting that synesthesia may be used as an aid to grapheme acquisition. Asano and Yokosawa (2013) hypothesized that the graphemecolor association for each grapheme is determined by the most distinctive feature for a particular grapheme (e.g., semantic information that is uniquely associated with that grapheme). According to their hypothesis, graphemes with different distinctive features tend to elicit corresponding synesthetic colors that are dissimilar to each other, and these resulting differences of synesthetic colors may contribute to the discrimination among the graphemes.

Research indicating the discriminating potential of synesthetic colors led us to hypothesize that the transfer of synesthetic grapheme-color association from familiar graphemes to novel ones (Mroczko et al., 2009; Rich et al., 2005; Witthoft \& Winawer, 2006) occurs only when synesthetic colors can demonstrate a discriminating potential. That is, a transfer emerges only when the members of a group of familiar graphemes, with each of which a novel grapheme is to be associated, elicit individually different synesthetic colors. Imagine a situation in which a grapheme-color synesthete first learns a group of novel graphemes, each of which is semantically/conceptually equivalent to one of the graphemes with which the synesthete is familiar, and the synesthetic colors of the entire group of the familiar graphemes happen to be similar to each other. In such a situation, if the transfer of synesthetic grapheme-color association from familiar graphemes to novel ones occurs, the synesthetic colors of the entire novel grapheme group would be similar to each other and hence would not facilitate the discrimination of novel graphemes. The discriminability hypothesis predicts that the transfer of synesthetic colors from semantically equivalent familiar graphemes would not occur in this situation; instead, the synesthetic colors of the novel graphemes may be determined by resources other than semantically equivalent familiar graphemes in order to make the synesthetic colors of a whole group of novel graphemes different from each other.

To test the discriminability hypothesis, we conducted an experiment in which Japanese grapheme-color synesthetes learned six novel graphemes (Thai characters) arbitrarily associated with familiar graphemes (Japanese Hiragana characters). Synesthetes were randomly assigned to one of two conditions: in the heterogeneous condition, the familiar graphemes elicited highly discriminable synesthetic colors, and in the homogeneous condition, the familiar graphemes elicited synesthetic colors that were less discriminable. If the mechanism suggested in the semantic hypothesis (Mroczko et al., 2009) and that suggested in the discriminability hypothesis, described above, work in a complementary fashion, then the synesthetic colors of the novel graphemes should be transferred from those of the semantically equivalent familiar graphemes when the synesthetic colors of a whole group of familiar graphemes are dissimilar, and would not be transferred when they are similar to each other. Therefore, it is predicted that less transfer of synesthetic colors to novel graphemes from the corresponding familiar graphemes should occur in the homogeneous condition than in the heterogeneous condition, with larger color distances between familiar and novel graphemes in the homogeneous condition.

\section{Method}

\section{Overview}

The experiment consisted of four sections: Pretest and Tasks 1,2 , and 3. In the web-based Pretest, we identified for each synesthete (1) synesthetic color categories elicited by each of a set of familiar Hiragana characters, and (2) novel Thai characters for which the synesthete did not experience synesthetic color at this time. Based on the results of the Pretest, we 
selected six familiar-novel grapheme pairs as stimuli for each synesthete. In the laboratory, each synesthete first selected synesthetic colors for the six familiar graphemes by using an RGB color palette (Task 1). The participant then learned the six novel-familiar grapheme pairs (Task 2). After the learning session, the participant performed the synesthetic color selection task for both familiar and novel graphemes (Task 3 ). The distances between the colors selected for the learned novel graphemes in Task 3 and those for the corresponding familiar graphemes in Task 1 were calculated as indicators of the degree of synesthetic color transfer.

\section{Participants}

Twenty-seven Japanese grapheme-color synesthetes (21 females, six males, mean age $=29.5$ years, $S D=10.0$ years) whose first language was Japanese participated in this study. All reported experiencing synesthetic colors when viewing Hiragana characters, as well as when viewing digits, letters of the English alphabet, and other types of Japanese characters (i.e., Katakana and Kanji characters). Participants were randomly assigned to either the heterogeneous $(n=13)$ or the homogeneous $(n=14)$ condition.

\section{Stimuli}

Among the entire set of 71 Hiragana characters, we used all the 46 basic characters as familiar graphemes. Hiragana is a Japanese script that represents vowels or syllables (i.e., combinations of a consonant and a vowel) and is typically mastered by Japanese people in childhood. We used the 42 consonant characters in current use in the Thai language as novel graphemes. These are orthographically dissimilar to Japanese and Latin graphemes and to Arabic digits, and Japanese people typically have low exposure to them in daily life.

\section{Procedure}

Each participant completed a web-based Pretest at a selfchosen location. Subsequently, the participant engaged in two computer-based tasks in a darkened room and one writing task under room light.

Pretest At some time before the day of the laboratory experiment, each participant completed a web-based Pretest (created on Google Forms) concerning their synesthetic experiences. The Pretest consisted of two sections. In the first section, participants selected synesthetic colors for each of the familiar Hiragana graphemes. On each trial, one of the 46 characters was presented at the top of the computer screen, and the participant chose one color term corresponding to the character from among 13 color terms frequently used by Japanese (Kuriki et al., 2017) meaning red, yellow, green, blue, pink, orange, brown, purple, light blue, yellow-green, white, black, and gray, respectively. They also rated how accurately each of the color terms they selected represented the synesthetic color they experienced, using four options: Accurate, Somewhat accurate, Not very accurate, The grapheme elicits only a weak sensation of a color in the first place. Each of the 46 characters was presented only once.

In the second section of the Pretest, participants were asked to indicate the degree of their sensation of synesthetic color when viewing the novel Thai graphemes. On each trial, one of the 42 characters was presented at the top of the screen, and the participant reported the color intensity it elicited using one of four options: Strong sensation of color, Slightly strong sensation of color, Little sensation of color, No color.

\section{Task 1: Synesthetic color measurement of familiar graphemes} On each trial of Task 1, each of the familiar graphemes (46 Hiragana basic characters) was presented on the top of a computer screen (Mitsubishi Diamondtron M2 RDF223G), and participants used a computer mouse to select a corresponding color from an RGB $256^{3}$ color palette displayed below it. If the presented character elicited no synesthetic color, they were asked to select a "no color" option. Stimulus characters were presented in random order, and no character was repeated.

Task 2: Learning session In the second task, participants learned a group of six novel Thai characters, each of which was arbitrarily associated with a different familiar Hiragana character. These associations were completely unrelated to the actual sounds of Thai characters. A different set of stimulus characters was selected for each participant based on the results of the Pretest. In the heterogeneous condition, the synesthetic colors for the six stimulus Hiragana characters were selected from six different color categories (i.e., color terms). In the homogeneous condition, on the other hand, all six stimulus Hiragana characters elicited synesthetic colors of the same category. In both conditions, Hiragana characters were selected from those for which the color terms were rated as "accurate" or "somewhat accurate" representations of the synesthetic colors, and Thai characters were selected from those eliciting ratings of "little sensation of color" or "no color." Further, we avoided including in the stimulus set Thai characters that were highly visually similar (see Fig. S1 in Supplementary Material 1).

The learning session consisted of the repetition of a sequence of events that included a handwriting section and a test section. This sequence is shown in Fig. 1. In the handwriting section, participants were first given a sheet of paper on which six pairs of familiar graphemes and novel graphemes were printed, and they wrote each pair of graphemes five times with a pencil. They then proceeded to the test section, which consisted of the following steps: They were first asked to write the novel grapheme that corresponded to each familiar 


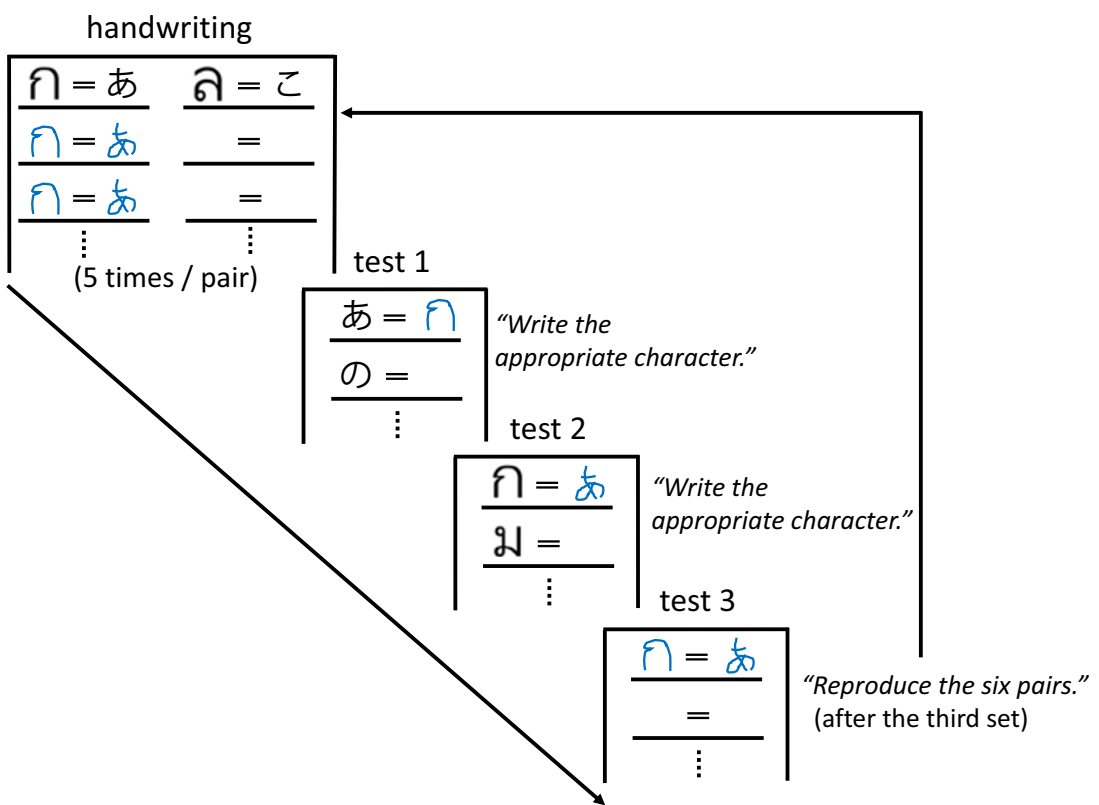

Fig. 1 Sequence of events in the learning session. Printed characters are shown in black and handwritten characters in blue. This sequence was

grapheme, and were then asked to write the familiar grapheme corresponding to each novel grapheme. After the participant repeated this three times and correctly reproduced all the graphemes on the last repeat, he or she was asked to recall the six pairs of novel and familiar graphemes. The learning session ended if the participant succeeded in recalling all pairs in succession; therefore each participant completed at least four sets of handwriting and test sections.

Task 3: Synesthetic color measurement for novel and familiar graphemes Participants selected a color corresponding to each novel grapheme (Thai character) and familiar grapheme (Hiragana basic character). All 42 Thai characters (including the six graphemes used in the learning session) and 46 Hiragana basic characters (including the six graphemes used in the learning session) were used as stimuli. The apparatus and procedure were the same as in Task 1.

Following the completion of Task 3, participants were asked whether there were Thai characters that elicited a synesthetic color at the beginning of Task 2 (i.e., the first time they saw Thai characters in the laboratory experiment).

\section{Results}

\section{Color consistency over time}

One participant who had experience of learning Thai characters was excluded from all analyses. In addition, two participants were excluded from all analyses because they reported having perceived specific color sensations for more than four repeated until the synesthete could correctly reproduce all six novelfamiliar grapheme pairs in succession

of the six novel Thai characters graphemes before they worked on the learning session.

Color coordinates in the CIE L*a*b* system, used in the following analyses, were converted from the CIE xyY coordinates of the presented colors; all were measured using a Topcon BM-7AC luminance colorimeter. We adopted the CIE L*a*b* system because it was designed in such a way that perceived color differences correspond to mathematical distances in all color ranges (Rothen, Seth, Witzel, \& Ward, 2013).

We first examined the consistency of participants' color selections over time to demonstrate that they were genuine synesthetes (Eagleman et al., 2007). The average of the color distances in the CIE L*a*b* system for the colors selected for a given Hiragana character in the two tasks (Task 1 and Task 3) was calculated for each participant. According to Rothen et al. (2013), the cut-off (maximum) value of color distance in the CIE L*a*b* color space that discriminates between synesthetes and non-synesthetes is 36.4. The average values of color distances for two participants were higher than the cut-off value (Rothen et al., 2013), indicating that the consistency of their color selections was not sufficient to regard them as synesthetes; thus, they were excluded from all subsequent analyses. Data from 22 participants (11 for each condition, see Supplementary Material 2) were thus analyzed in the next section.

\section{Occurrence probability of synesthetic colors for novel characters}

To investigate whether the learning session induced the experience of synesthetic colors for novel characters, we calculated 
the probability of occurrence of synesthetic colors for novel characters. We divided the number of learned Thai characters that elicited a synesthetic color in Task 3 (i.e., after the learning session, $\max =6$ ) by the number of learned Thai characters. If there were any Thai characters reported to have had elicited a synesthetic color at the beginning of the learning session, they were excluded from the analysis. This probability was $83.3 \%$ ( $S E=9.4 \%$ ) for participants in the heterogeneous condition $(n=11)$, and $79.5 \%(S E=9.9 \%)$ for participants in homogeneous condition $(n=11)$. The occurrence probability of synesthetic colors did not different significantly between the two conditions, $t(20)=0.29, p=.78$.

The purpose of the current study was to examine whether the discriminating potential of synesthetic colors modulates the degree of transfer of synesthetic color from familiar to novel graphemes. We can examine the hypothesis only for cases in which synesthetes experienced synesthetic colors for novel graphemes after (but not before) learning the graphemes. For this reason, we excluded data from three participants whose occurrence probability was below $50 \%$ (two participants in the heterogeneous condition and one participant in the homogeneous condition). Data from nine participants in the heterogeneous condition and ten participants in the homogeneous condition were analyzed in the subsequent analyses.

\section{Color consistency between familiar and novel graphemes}

As an indicator of the degree of synesthetic color transfer, average color distance in the CIE L*a*b* system between the colors selected for learned novel graphemes in Task 3 and those for the corresponding familiar graphemes in Task 1 were calculated. As in the previous analysis, if any Thai characters were reported to have had elicited a synesthetic color at the beginning of the learning session, these characters were excluded from the analysis. Representative synesthetic color examples are shown in Fig. 2, and two histograms of all paired color distances in the heterogeneous and homogeneous conditions are shown in Fig. S2 in Supplementary Material 1. In the heterogeneous condition, the color distances for most color pairs fell within the range of 0 to 10 in the CIE L*a*b* system (i.e., the colors were approximately the same), and the probability decreased as the color distance increased. In the homogeneous condition, on the other hand, the probability was uniformly distributed. A linear mixed model predicted color distances for colors selected for familiar and novel graphemes, including condition (heterogeneous or homogeneous) as predictors and a random intercept to account for the random variation in participants. Overall, condition was a significant predictor of color consistency between familiar and novel graphemes $(t=2.56, p=.021)$ : Color distances were significantly smaller for participants in the heterogeneous condition than for those in the homogeneous condition (see Fig. 3), indicating that novel graphemes in the heterogeneous condition began to elicit synesthetic colors that were more like those elicited by corresponding familiar graphemes.

\section{Did the association between colors and novel graphemes remain stable over time?}

Following the results noted above, we conducted an additional experiment to investigate whether the associations between colors and novel graphemes remain over time in each condition. Five participants in the heterogeneous condition and five in the homogeneous condition participated in the experiment after 1-2 years of Task 1-3. They selected a color corresponding to each Thai character (if any). Results indicated that, regardless of the conditions, about half of the Thai characters that had been presented in the learning session and had elicited specific synesthetic colors in Task 3 no longer elicited color. Moreover, the colors selected for the other half of the characters were not consistent with those selected for the same characters in Task 3, indicating that the association between colors and novel graphemes did not remain stable over 1-2 years in either condition. A more detailed description of the additional experiment can be found in Supplementary Material 1.

\section{Discussion}

Synesthetic color similarity between familiar graphemes (Hiragana characters) and novel graphemes (Thai characters) was greater when the familiar graphemes elicited highly discriminable synesthetic colors from one another, although the occurrence probability of synesthetic colors for novel graphemes did not significantly differ between heterogeneous and homogeneous conditions. The transfer of synesthetic grapheme-color association to novel graphemes (Mroczko et al., 2009; Rich et al., 2005; Witthoft \& Winawer, 2006) occurred only when synesthetic colors functioned as discriminating markers. This pattern of results is a new demonstration of the effect of the heterogeneity of synesthetic colors for familiar graphemes on the association of synesthetic colors with novel graphemes associated with these familiar graphemes. Moreover, it also suggests that the transfer of synesthetic colors can be explained not only by the semantic hypothesis (Mroczko et al., 2009) but also by the discriminability hypothesis. However, it should be noted that the heterogeneity of synesthetic colors for familiar graphemes does not appear to produce a significant effect on the long-term retention of the synesthetic colors for novel graphemes.

The occurrence probability of synesthetic colors was generally high $(79.5 \%)$ regardless of condition. The result is consistent with prior research showing that the synesthetic association for a novel grapheme can be acquired via grapheme learning even in adulthood (Mroczko et al., 2009; Rich et al., 
Heterogeneous condition
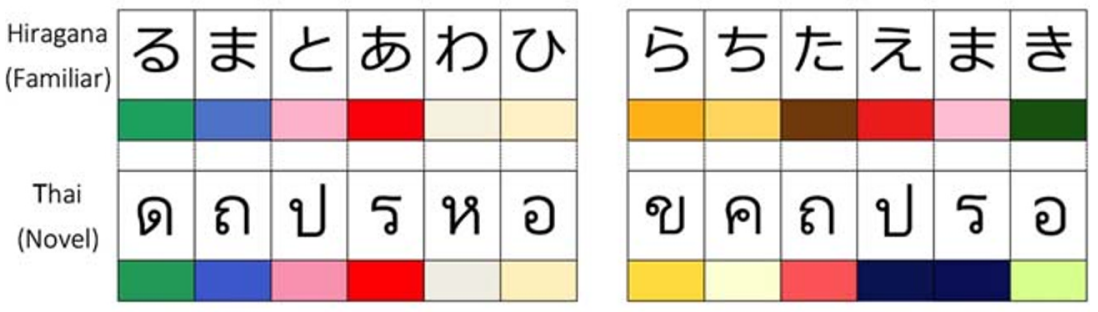

Homogeneous condition
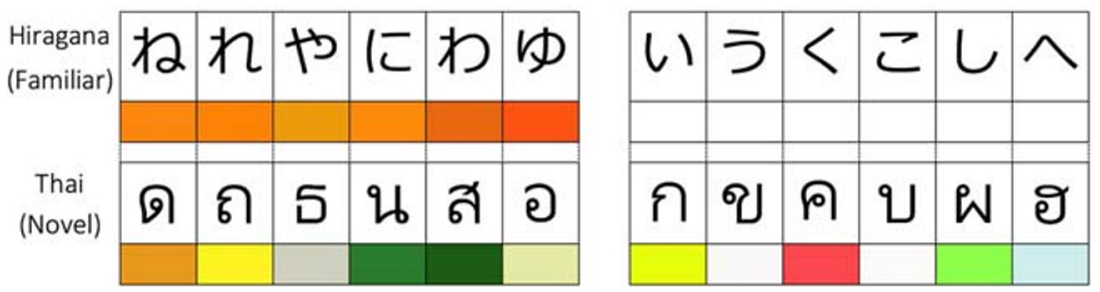

Fig. 2 Representative synesthetic color examples. Examples for four synesthetes (two each in heterogeneous and homogeneous conditions) are shown. All selected colors for 22 participants who were regarded as genuine synesthetes are shown in Supplementary Material 2
2005; Witthoft \& Winawer, 2006). However, in the present homogeneous condition, novel graphemes induced synesthetic colors that were dissimilar from the synesthetic colors elicited by the associated familiar graphemes. Although our results do not provide grounds for identifying the source of the synesthetic colors elicited by novel graphemes, it is reasonable to propose that those synesthetic colors associated with other familiar graphemes transferred to the novel graphemes. Given that no grapheme information was provided for a novel grapheme (a Thai character) other than that of the associated familiar grapheme (a Hiragana character) in our experiment, it seems more likely that the synesthetic color of the novel grapheme was transferred from another familiar grapheme whose visual shape (orthography) is similar to that of the novel grapheme. Witthoft and Winawer (2006) have found

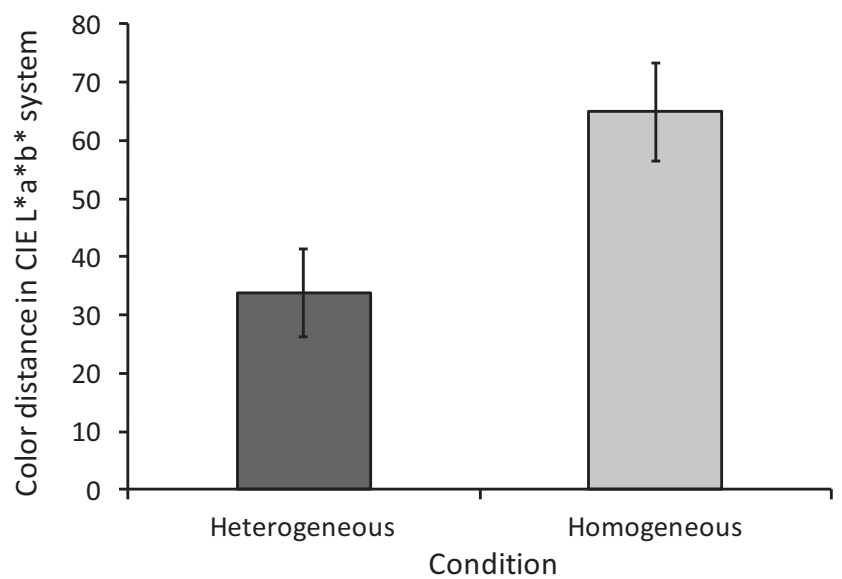

Fig. 3 Average color distances in the CIE L*a*b* system for colors selected for Hiragana characters in Task 1 and the corresponding Thai characters for each condition. Error bars represent standard errors that the synesthetic association between a familiar grapheme (e.g., the letter "R") and a specific color can be transferred to a novel grapheme that is visually similar to the familiar grapheme (e.g., the Cyrillic "Ya", Я) although they do not share phonetic information. Based on the finding of Witthoft and Winawer, the synesthetic colors of novel graphemes that were unlike those of familiar graphemes may have been the consequence of the transfer of synesthetic associations with other familiar graphemes that partly shared information (especially orthographical information) with the novel graphemes.

Previous studies of grapheme-color synesthesia have shown that multiple grapheme properties (e.g., sounds, meanings, orthography, ordinality, and so on) determine synesthetic colors; further, the process of grapheme learning strongly affects grapheme-color associations (Asano \& Yokosawa, 2013; Rich et al., 2005; Simner, 2007). Consistent with this view, Mroczko et al. (2009), who proposed the semantic hypothesis, showed that a synesthetic color associated with a grapheme can be automatically transferred to a novel grapheme as soon as the synesthete learns that the novel grapheme is semantically/conceptually equivalent to the familiar one. Our results, which support the discriminability hypothesis, add new insight to their findings. Specifically, synesthetic associations between graphemes and colors may be affected not only by properties of the graphemes (the inducers) but also by the properties of the colors (the concurrents), especially by their "discriminating" potential (Asano \& Yokosawa, 2013). In the present study, the discriminability of synesthetic colors affected the synesthetic associations between graphemes and colors, directly supporting the hypothesis that "synesthetic colors function as discriminating markers of graphemes during learning graphemes" (Asano \& Yokosawa, 2013). This is 
consistent with the suggestion that synesthesia can be used as a grapheme acquisition aid and can help induce learning (Watson et al., 2014, 2017).

Author Note This research was supported in part by KAKENHI (Grant-in-Aid for Scientific Research (B) \#19H01770 and \#26285164) awarded to Kazuhiko Yokosawa.

\section{References}

Asano, M., \& Yokosawa, K. (2012). Synesthetic colors for Japanese late acquired graphemes. Consciousness and Cognition, 21, 983-993.

Asano, M., \& Yokosawa, K. (2013). Grapheme learning and graphemecolor synesthesia: Toward a comprehensive model of graphemecolor association. Frontiers in Human Neuroscience, 7:757. doi: https://doi.org/10.3389/fnhum.2013.00757

Dixon, M. J., Smilek, D., Duffy, P. L., Zanna, M. P., \& Merikle, P. M. (2006). The role of meaning in grapheme-colour synaesthesia. Cortex, 42, 243-252.

Eagleman, D. M., Kagan, A. D., Nelson, S. S., Sagaram, D., \& Sarma, A. K. (2007). A standardized test battery for the study of synesthesia. Journal of Neuroscience Methods, 159, 139-145.

Kuriki, I., Lange, R., Muto, Y., Brown, A. M., Fukuda, K., Tokunaga, R., Delwin, T., Lindsey, D. T., Uchikawa, K., \& Shioiri, S. (2017). The modern Japanese color lexicon. Journal of Vision, 17(3), 1. doi: https://doi.org/10.1167/17.3.1

Mroczko, A., Metzinger, T., Singer, W., \& Nikolić, D. (2009). Immediate transfer of synesthesia to a novel inducer. Journal of Vision, 9(12), 25. doi:https://doi.org/10.1167/9.12.25

Rich, A. N., Bradshaw, J. L., \& Mattingley, J. B. (2005). A systematic, large-scale study of synaesthesia: Implications for the role of early experience in lexical-colour associations. Cognition, 98, 53-84.

Rothen, N., Seth, A. K., Witzel, C., \& Ward, J. (2013). Diagnosing synaesthesia with online colour pickers: Maximising sensitivity and specificity. Journal of Neuroscience Methods, 215, 156-160.

Simner, J. (2007). Beyond perception: Synaesthesia as a psycholinguistic phenomenon. Trends in Cognitive Sciences, 11, 23-29.

Watson, M. R., Akins, K. A., Spiker, C., Crawford, L., \& Enns, J. T. (2014). Synesthesia and learning: a critical review and novel theory. Frontiers in Human Neuroscience, 8:98. doi:https://doi.org/10. 3389/fnhum.2014.00098

Watson, M. R., Chromý, J., Crawford, L., Eagleman, D. M., Enns, J. T., \& Akins, K. A. (2017). The prevalence of synaesthesia depends on early language learning. Consciousness and Cognition, 48, 212231.

Witthoft, N., \& Winawer, J. (2006). Synesthetic colors determined by having colored refrigerator magnets in childhood. Cortex, 42, $175-183$.

Publisher's note Springer Nature remains neutral with regard to jurisdictional claims in published maps and institutional affiliations. 